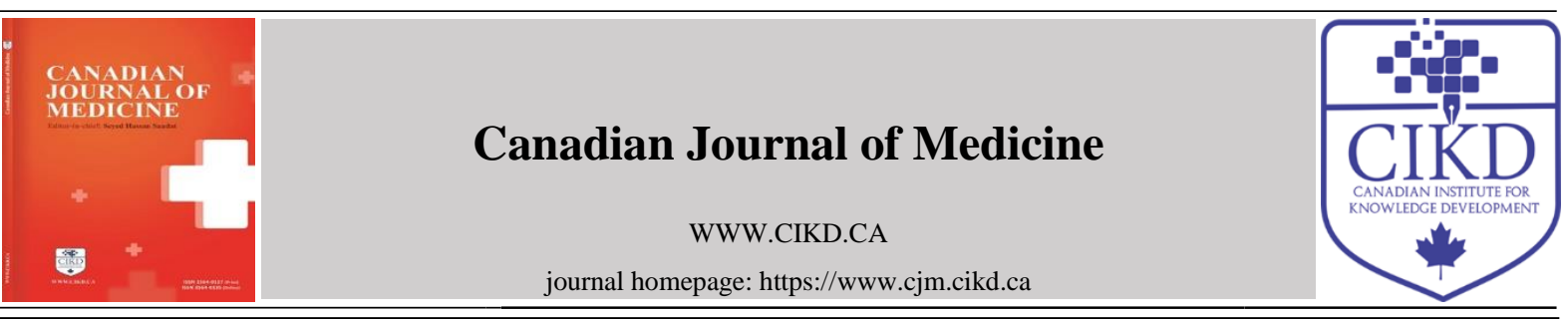

\title{
Pitfalls in Interpretation of SARS-CoV-2 Infection-related Neurological Complications
}

\author{
Seraph Shi Kei Wu ${ }^{1}$, Sunny Chi Lik $\mathrm{Au}^{2^{*}}$ \\ ${ }^{1}$ LKS Faculty of Medicine, University of Hong Kong, Hong Kong, China \\ ${ }^{2}$ Department of Ophthalmology, Tung Wah Eastern Hospital, Hong Kong, China
}

\begin{tabular}{l} 
Keywords: \\
COVID-19, SARS-CoV-2, \\
Meta-Analysis, Systematic \\
Review, neurology \\
\hline
\end{tabular}

Received

07 August 2021

Received in revised form

22 October 2021

Accepted

26 October 2021

*Correspondence:

kilihcua@gmail.com

\section{Dear Editor,}

Coronavirus disease (COVID-19) is an ongoing global pandemic caused by severe acute respiratory syndrome coronavirus 2 (SARS-CoV-2). Many systematic reviews and metaanalyses discussed the correlation of COVID-19 with a different disease. Given the urgent need for data, some meta-analyses containing datasets included many manuscripts, but their providence was not clearly reported. The possible overlap between some of the studies included in the analyses is a significant issue for conducting systematic reviews during the COVID-19 pandemic.

(C)CIKD Publishing

Coronavirus disease (COVID-19) is an ongoing global pandemic caused by severe acute respiratory syndrome coronavirus 2 (SARS-CoV-2). Many systematic reviews and metaanalyses discussed the correlation of COVID-19 with a different disease. Given the urgent need for data, some meta-analyses containing datasets included many manuscripts, but their providence was not clearly reported. The possible overlap between some of the studies included in the analyses is a significant issue for conducting systematic reviews during the COVID-19 
pandemic. Here, we randomly selected two meta-analyses in the field of neurology and COVID-19 to illustrate the situation. One meta-analysis is on pediatric patients, whereas the other was conducted on adult subjects. Hopefully, this arrangement will provide a more comprehensive view on the difficulties encountered by researchers when studying different COVID-19 infected age populations.

Pediatric study was a systematic review and meta-analysis on neurological complications in COVID-19 children [1]. Preliminary studies in the analysis showed possible bias due to including overlapped COVID-19 pediatric subjects. One of the principles of meta-analysis is that correlated data should not be included; duplicate publication bias can potentially skew the final outcome of the meta-analysis [2, 3, 4]. Multiple included studies were conducted at the same Wuhan research site of Children's Hospital $[5,6,7,8,9,10]$. As described by primary studies, Wuhan Children's Hospital was the only designated hospital for treating young COVID-19 patients assigned by the central government in Wuhan, China. This statement was consistently echoed throughout the methodology part of the articles published by Li et al. [5] Lu et al. [6] Li et al. [7] Sun et al. [8] Wu et al. [9] Xia et al. [10] Ma et al. [11]. A definite pediatric age group is overlapping for the meta-analysis of included subjects from reviewing the age range column in Table 1 of Panda et al.'s meta-analysis [1]. Besides, the recruitment dates of different primary studies showed overlapping when going through them one by one. From our tidying up of data, all of the above-mentioned studies recruited pediatric COVID-19 subjects hospitalized from 23 January 2020 to 8 February 2020, despite these studies were of different start and end dates.

There is a saying across the academic medical research and statistics arena of "Garbage in, Garbage out" [12], which is particularly an important limitation if it happened on a metaanalysis $[13,14]$. Moving into the adult study, it was a systematic review and meta-analysis on the incidence of acute cerebrovascular disease in COVID-19 patients [15]. A primary study by Li et al. [16] reported that "Part of analysis results of 214 patients with COVID-19 had been published in our previous paper on JAMA Neurology [17]. In the study, the analysis results of 219 patients were derived completely from different perspectives ...". Reviewing the methodology of the two publications $[16,17]$ demonstrated that both studies were recruiting patients with confirmed COVID-19 admitted to the Union Hospital of Huazhong University of Science and Technology between 16 January and 19 February 2020. The possible bias due to overlap of 214 COVID-19 patients cannot be ignored in studies performed over a period of time and in one place [17]. These duplicated subjects would have skewed the pooled analysis in Figure 1 of Nannoni et al's work [15], and part of the subsequent summed up data in the subgroup analyses involving both studies by Li et al. and Mao et al. [15,16,17]

Au et al. also pointed out similar pitfalls in another meta-analysis involving stroke among COVID-19 subjects [18]. Despite the possible overlap in patients we identified, the pooled results in the original analysis did not defer greatly from our recalculation of the revised analyses. Thus, readers need to be cautious in interpreting the meta-analysis's results when there is potential duplicate publication bias. Authors should try their best to avoid including potential duplicate subjects by carefully looking into each primary study's research site and possible overlapping time period of subject recruitment. 


\section{References}

[1] Panda PK, Sharawat IK, Panda P, et al. Neurological complications of SARS-CoV-2 infection in children: A systematic review and meta-analysis [published online ahead of print, 2020 Sep 10]. J Trop Pediatr. 2020; fmaa070.

[2] Tramèr MR, Reynolds DJ, Moore RA, et al. Impact of covert duplicate publication on meta-analysis: a case study. BMJ. 1997;315(7109):635-640.

[3] Choi WS, Song SW, Ock SM, et al. Duplicate publication of articles used in meta-analysis in Korea. Springerplus. 2014;3:182.

[4] von Elm E, Poglia G, Walder B, et al. Different patterns of duplicate publication: an analysis of articles used in systematic reviews. JAMA. 2004;291(8):974-80.

[5] Li H, Chen K, Liu M, et al. The profile of peripheral blood lymphocyte subsets and serum cytokines in children with 2019 novel coronavirus pneumonia. J Infect 2020;81:115-20.

[6] Lu X, Zhang L, Du H, et al. SARS-CoV-2 infection in children. N Engl J Med 2020; 382:1663-5.

[7] Li Y, Wang H, Wang F, et al. Comparison of hospitalized patients with pneumonia caused by COVID-19 and influenza A in children under 5 years. Int J Infect Dis 2020;98:80-3.

[8] Sun D, Chen X, Li H, et al. SARS-CoV-2 infection in infants under 1 year of age in Wuhan City, China. World J Pediatr 2020;16:260-6.

[9] Wu H, Zhu H, Yuan C, et al. Clinical and immune features of hospitalized pediatric patients with coronavirus disease 2019 (COVID-19) in Wuhan, China. JAMA Netw Open 2020; $3:$ e2010895.

[10] Xia W, Shao J, Guo Y, et al. Clinical and CT features in pediatric patients with COVID-19 infection: different points from adults. Pediatr Pulmonol 2020;55:1169-74.

[11] Ma H, Hu J, Tian J, et al. A single-center, retrospective study of COVID-19 features in children: a descriptive investigation. BMC Med 2020;18:123.

[12] Kim Y, Huang J, Emery S. Garbage in, garbage out: Data collection, quality assessment and reporting standards for social media data use in health research, infodemiology and digital disease detection. J Med Internet Res. 2016;18(2):e41.

[13] Lee YH. An overview of meta-analysis for clinicians. Korean J Intern Med. 2018;33(2):277-283.

[14] Smith TO, Hing CB. "Garbage in, garbage out"- the importance of detailing methodological reasoning in orthopaedic meta-analysis. Int Orthop. 2011;35(2):301-302.

[15] Nannoni S, de Groot R, Bell S, Markus HS. Stroke in COVID-19: A systematic review and meta-analysis. Int J Stroke. 2021; 16(2):137-149

[16] Li Y, Li M, Wang M, Zhou Y, Chang J, Xian Y, Wang D, Mao L, Jin H, Hu B. Acute cerebrovascular disease following COVID-19: a single center, retrospective, observational study. Stroke Vasc Neurol. 2020;5(3):279-284.

[17] Mao L, Jin H, Wang M, Hu Y, Chen S, He Q, Chang J, Hong C, Zhou Y, Wang D, Miao X, Li Y, Hu B. Neurologic manifestations of hospitalized patients with coronavirus disease 2019 in Wuhan, China. JAMA Neurol. 2020; 77(6):683690.

[18] Au SCL. Comments on Stroke as a Neurological Complication of COVID-19: A systematic review and meta-analysis of incidence, outcomes and predictors [published online ahead of print]. J Stroke Cerebrovasc Dis. 2021. doi:10.1016/j.jstrokecerebrovasdis.2021.105863

\section{Acknowledgements}

Not applicable.

\section{Disclosure Statement}

No potential conflict of interest was reported by the authors.

\section{Funding Acknowledgements}

Not applicable. 